EGU2020-21041, updated on 17 Jun 2021

https://doi.org/10.5194/egusphere-egu2020-21041

EGU General Assembly 2020

(c) Author(s) 2021. This work is distributed under

the Creative Commons Attribution 4.0 License.

\title{
Characterisation of East Siberian paleodiversity based on ancient DNA analyses of the Batagay megaslump exposure
}

\author{
Jeremy Courtin ${ }^{1}$, Amedea Perfumo ${ }^{1}$, Kathleen Stoof-Leichsenring ${ }^{1}$, and Ulrike Herzschuh ${ }^{1,2,3}$ \\ ${ }^{1}$ Alfred-Wegener-Institute Helmholtz-Center for Polar and Marine Research, Polar Terrestrial Environmental Systems, \\ Potsdam, Germany \\ ${ }^{2}$ Institute of Biology and Biochemistry, University of Potsdam, Potsdam-Golm, Germany \\ ${ }^{3}$ Institute of Earth and Environmental Science, University of Potsdam, Potsdam-Golm, Germany
}

With the ongoing Arctic warming, permafrost thaw accelerated during the last decade as much as it is now a global concern for biodiversity loss, food webs and biogeochemical cycling. This rapid permafrost degradation forms features such as massive retrogressive thaw slumps that give access to exceptional records for Quaternary biodiversity change investigations. The Batagay megaslump located in northern Yakutia, East Siberia, is the world's largest thawslump known to date, and along its $\sim 55 \mathrm{~m}$ high headwall it gives access to Late and Mid Pleistocene permafrost deposits up to more than 500 kyrs in age. During an expedition to this unique site in 2017, sediment samples were collected with ages from more than 500 kyrs to modern time for the analysis of ancient DNA (aDNA). Our aim is to characterise the biodiversity and changes over geological timescales of this region in East Siberia. Using the aDNA extracted from these ancient environmental samples, we first performed a metabarcoding analysis (chloroplast trnL) to investigate past vegetation composition. We then performed a shotgun metagenomic analysis, which enabled a much higher depth of sequence data and allowed us to access the entire biodiversity, from Eukaryotes to Prokaryotes, Archaea and Viruses. This approach opened up new horizons, making it possible not only to investigate biodiversity composition and changes but also to infer on potential interactions across taxa and kingdoms. Both methods together allowed comparison and ensured robustness of the results obtained. We present here one of the very first studies done on the global, past and modern, biodiversity of permafrost regions which holds an enormous potential to reveal new insights into the evolution of this fragile ecosystem. 